\title{
Soundproofing presses by placing its nodes in a soundproof casing, including drive components
}

\author{
Sergei Pushenko ${ }^{1}$, Evgeny Bozhko ${ }^{1}$, Irina Zolotuhina, ${ }^{1, *}$ \\ ${ }^{1}$ Don State Technical University, 1, pl. Gagarina, 344002, Rostov-on-Don, Russia
}

\begin{abstract}
Noise is one of the most unfavorable environmental factors in production facilities, for which it is most difficult to achieve compliance with sanitary standards. Prolonged exposure to intense vibrations and noise leads to noise and vibration diseases. The effect of noise on the human body is not limited to exposure to the organ of hearing. Irritation by noise is transmitted to the central and autonomic nervous systems, through them acts on the internal organs, leading to significant changes in the functional state of the organism. With pulsed and irregular noise, the degree of impact of noise on the body of workers increases. Most types of forging equipment relate to impact machines, during the operation of which impulse noise occurs, the levels of which at workplaces, in most cases, exceed the maximum permissible values and relate to those factors, the significance of which in some cases is greater than dustiness, high temperature, manipulation of heated workpieces. Therefore, the problem of reducing vibration and noise in the forge shops is extremely relevant and has great scientific and socio-economic significance. To solve the problem of noise reduction in forge shops, various options for reducing the noise activity of equipment have been developed/ It is possible to most effectively reduce noise by changing the material and shapes of the beds, noise-attenuating casings, oil baths for gears.
\end{abstract}

\section{Introduction}

All these measures on existing and running equipment are implemented through the use of passive noise protection measures, which in the current production are little feasible, and in most cases impossible at all. The problem of reducing noise in the source of its occurrence and bringing the noise levels to the maximum permissible values should be solved at the design stage. Such an approach to solving the problem of noise reduction is the most justified both from the scientific, technical and economic points of view. Work in this direction is carried out in far abroad countries and is almost completely absent in our country. This is explained, first of all, by the absence of reliable engineering methods for calculating noise and vibration, which would take into account the design features of forging machines and technological operating modes, made it possible to assess the expected noise levels at the stage of calculation and design and choose engineering

*Correspondimg author: 5976765@mail.ru 
solutions to reduce noise levels up to standard values for each specific type of forging equipment.

When working in conditions of vibration, labor productivity decreases, the number of injuries increases. At some workplaces in agricultural production, vibrations exceed normalized values, and in some cases they are close to limit. The vibration levels on the controls do not always comply with the standards. Typically, low-frequency vibrations that negatively affect the body predominate in the vibration spectrum. Some types of vibration adversely affect the nervous and cardiovascular systems, the vestibular apparatus. The most harmful effect on the human body is provided by vibration, the frequency of which coincides with the frequency of natural vibrations of individual organs, the approximate values of which are as follows $(\mathrm{Hz})$ : stomach $-2 \ldots 3$; kidneys $-6 \ldots 8$; heart $-4 \ldots 6$; intestines $-2 \ldots 4$; vestibular apparatus - 0.5 ..L, 3; eyes $-40 \ldots 100$, etc.

The effect on muscular reflexes reaches $20 \mathrm{~Hz}$; the tractor-loaded seat on the tractor has its own vibration frequency of $1.5 \ldots 1.8 \mathrm{~Hz}$, and the rear wheels of the tractor $-4 \mathrm{~Hz}$. The vibration is transmitted to the human body at the moment of contact with the vibrating object: when acting on the limbs, local vibration occurs, and on the whole body - general. Local vibration affects neuromuscular tissues and the musculoskeletal system and leads to spasms of peripheral vessels. With prolonged and intense vibrations, in some cases, a professional pathology develops (local vibration often leads to it): peripheral, cerebral or cerebral-peripheral vibrational disease. In the latter case, there are changes in cardiac activity, general excitement, or, conversely, inhibition, fatigue, the appearance of pain, a feeling of shaking of the internal organs, nausea. In these cases, vibrations also affect the osteoarticular apparatus, muscles, peripheral circulation, vision, hearing $[1,3,5,7,9]$. Local vibrations cause spasms of blood vessels that develop from the terminal phalanges of the fingers, spreading to the entire hand, forearm, and cover the vessels of the heart.

Workers can be exposed to occupational vibration through the use of power or pneumatic hand tools or other machinery, or by driving large transportation, construction or agricultural vehicles. Vibration that is generated through the use of powered hand tools, and is transmitted from the tool to the hand-arm system is referred to as hand-transmitted vibration. However, recent studies have also demonstrated that vibration can be transmitted through platforms workers are standing on, and in these situations, the point of contact is the feet. Workers can also be exposed to whole body vibration (WBV). WBV exposure occurs in occupations where workers are driving trucks, large earth moving vehicles, or where they are using hand tools where the amplitude of the vibration is great enough to be transmitted to other portions of the body, such as in workers using jack-hammers.

Exposure to WBV is of concern within the workforce because it's associated with the development of a number of negative health consequences including back and neck pain, and potentially, cardiovascular disease, the development of various neuropathies, digestive problems, headaches, dizziness, motion sickness and possibly cancer. However, workers exposed to WBV are often also exposed to a number of other risk factors that may contribute to the development of these negative health effects. These risk factors include maintaining a static posture for a long-period of time, torque or twisting of the abdomen to view the area around the vehicle, and heavy lifting that often occurs when a vehicle is being loaded or unloaded. In addition to vibration and the physical exposures associated with a job, there may be other co-exposures to chemicals or certain environmental conditions that contribute to the development of disease or injury in workers. Because most workers are exposed to multiple factors that may induced injury or illness, it's difficult to determine which factors pose the greatest risk for inducing injury or illness. Experimental studies, examining the effects of each of these factor individually on health outcomes can provide additional information that will help determine the contribution of each exposure factor to various health problems. 
This review will describe the industrial sectors where vibration exposure is most prevalent and the health effects associated with exposure to HTV and WBV. Experimental methods used to measure and characterize vibration generated in various occupational settings are discussed, and models that have been used to uncover the etiology of vibrationinduced injuries. Although numerous studies have been published on both HTV and HBV, there are new epidemiological studies showing an increased risk of specific cancers with exposure to WBV. Therefore additional etiological studies need to be performed. New avenues for research are discussed below.

\section{Methods}

The authors selected a sliding casing design in the form of a parallelepiped. The dimensions are chosen so that the distance between the machine and the inner surface is as small as possible during normal operation with the casing closed. According to the authors, the installation of the casing led to the achievement of sanitary standards.

Noises arising from the operation of presses are divided into mechanical and aerodynamic. The sources of mechanical noise include sound vibrations of the bed and other massive parts of the press, resulting from numerous shocks at all links of the kinematic chains and in the working area during idle and working press. In addition, noise is created when falling and moving workpieces and finished parts through transporting devices in containers. Aerodynamic noise occurs when the clutch and brake are turned on when the scale is blown off from the finished products of the tool and from the work of the fan for blowing jobs (fig.1).

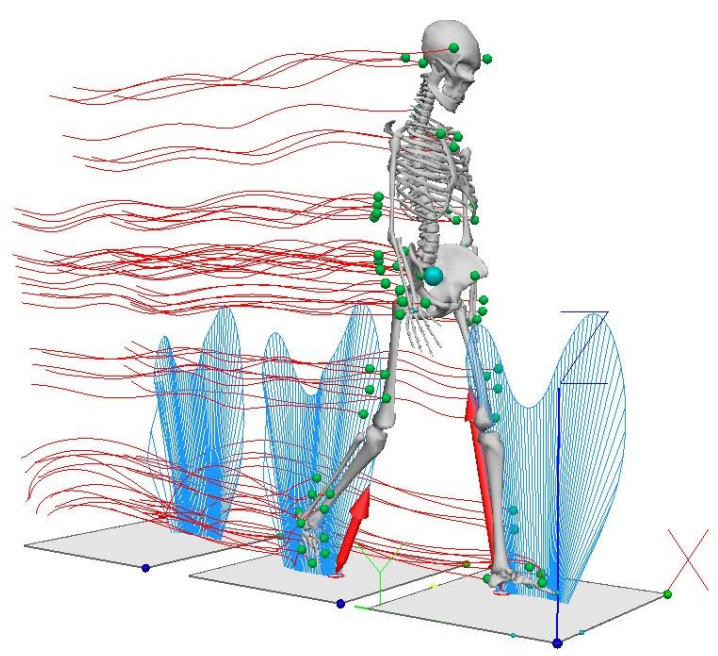

Fig. 1. Vibration effects.

To reduce mechanical noise, it is necessary to reduce the force and speed of impact of parts, replace impact interaction by shockless, reduce the number of revolutions of rotating masses, increase the accuracy of manufacturing parts and press assemblies, apply lubrication to mutually moving parts, make one or both of the colliding parts from a material with a lower elastic modulus, to cover colliding elements with non-metallic materials, to ensure careful static and dynamic balancing of moving parts $[1,3,5]$. 
The strength and speed of impact of parts can be reduced by installing knee-lever or articulated drive mechanisms that provide the differential speed of the press slide on different parts of the working stroke.

Shuler (Germany) installs a drive on punching machines that allows reducing noise by $65 \%$ shortly before contact with the workpiece, and clutch and brake hydraulic control on eccentric presses, reducing the slide speed $100 \mathrm{~mm}$ to bottom dead center (press noise reduced to $80 \mathrm{dBA}$ ). The noise level of the hydraulic presses of the Expulsor series of the SHP6300 and SHP1000 models, manufactured by Tissen Maschinen (Germany), is reduced from 100-105 dBA to $75 \mathrm{dBA}$, due to the servo-control equipment that allows you to program the position of the slider as a function of time and acting on an electronic signal. The stamping machine manufactured by the company Dansk pressfabrik (Denmark), which is equipped with an electro-hydraulic system that provides adjustment of the stroke in the range from 9 to $100 \mathrm{~mm}$, allows to reduce noise during stamping.

The noise level of the press also depends on its service life and the quality of the repair. Noise levels of working presses are on average 5-6 dBA lower. Reducing the noise level after repair provides increased accuracy of gears and mounting dimensions of the gearbox, reduced gaps in the actuator by improving the accuracy of manufacturing of mating parts. For example, reducing the clearance in a ball screw pair equal to $0.7 \mathrm{~mm}$ and the clearance between the pins and bearings equal to 0.004 of their diameter reduces the noise of the press by 20 tf by $4-20 \mathrm{dBA}$.

With an increase in the rotational speed of the crankshaft of the press, the noise level increases by about 3-4 dBA for every ten strokes or by $9 \mathrm{dBA}$ when doubling the speed. So, for example, the noise level of a grooved machine at 1100 stroke / min-99 dBA, and at 400 stroke / min-92 dBA.

Beds and gears are recommended to be cast from cast iron with spheroidal graphite, since their noise is less than that of welded beds and gears with equal mass. Replacing spur gears with helical gears or chevron gears reduces drive noise when idling by $8 \mathrm{dBA}[2,4,6]$.

Komatzy Maypress manufactures a 300 tf two-post press with a lower knee lever drive, the switching noise level of which is reduced from $86-88$ to $76-78 \mathrm{dBA}$ due to the installation of the clutch-brake unit in an oil bath.

The sound level created by a hydraulic press with a force of $100 \mathrm{tf}$ when installing pumps on the press head is $85 \mathrm{dBA}$ at idle and 90-92 dBA at full load, and when installing pumps under the floor, respectively 80 and $85 \mathrm{dBA}$. Another way to reduce noise is to use double-stand presses instead of C-type presses, which provides, for example, noise reduction from 100-104 $\mathrm{dBA}$ for presses with a force of 25-200 tf with an open bed to 90 dBA for two rack presses (fig.1). 

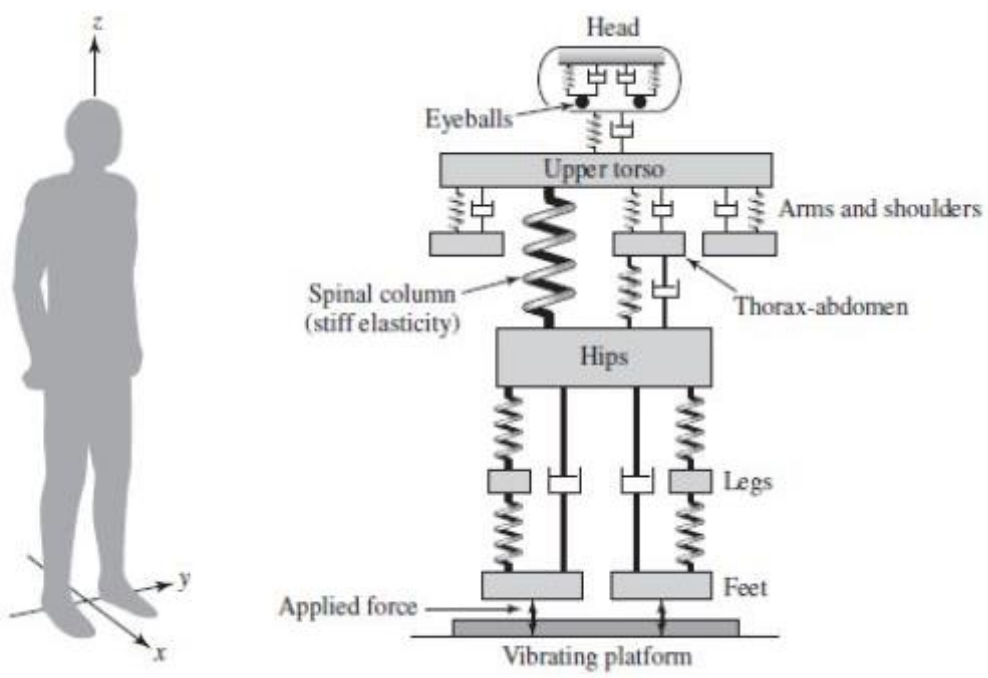

Fig. 2. Human vibration pattern.

On punching presses, the highest noise level occurs at the time of separation of the workpiece material. When punching small holes, it is recommended to use presses with a force significantly greater than the calculated one, and when punching large parts, try to reduce the amount of required force. The press can be equipped with a device to reduce the noise level, by using which the kinetic energy of the press frame and dies at the end of the stroke gradually decreases to zero, reducing the noise caused by vibration during unloading of the frame. For a press with a force of 35 ton-force, the noise level reduction during installation is 15-20 dBA. The device contains a belt brake mounted on the crankshaft of the press, and a cam that pulls the brake band about $5^{\circ}$ to bottom dead center $[7,9,11]$.

\section{Results}

The most effective measure is the soundproofing of the press by placing the most noisy nodes in a soundproof casing or a common casing device, or a press chamber. Closing the press drive clutch with easily removable covers made of steel sheet or plywood with a coating of the internal surface with sound-absorbing material reduces noise by 4-5 dBA. Effectively fencing dies with transparent polyvinyl chloride screens or placing dies in a casing with air in and out so that the workpiece is deformed in a stream of compressed air. Placing the press processing area in a casing with a sound-absorbing coating reduces noise by $8 \mathrm{dBA}[8,10,12]$. 


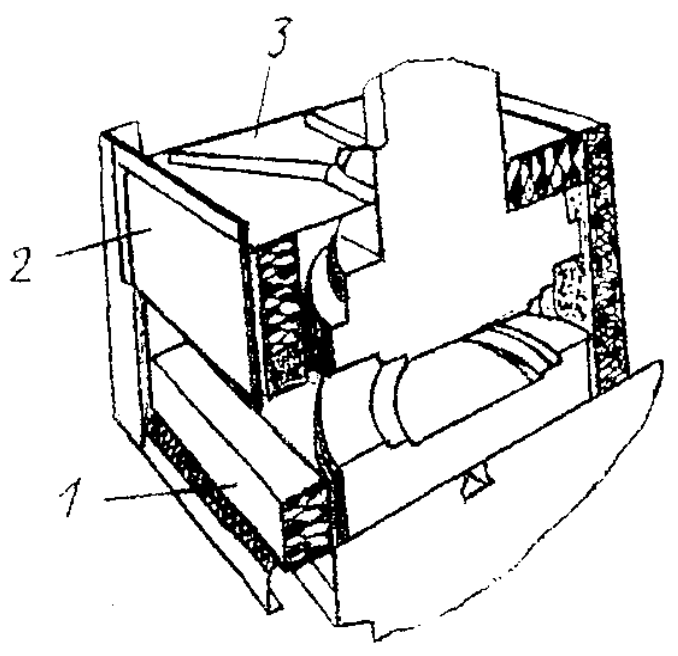

Fig. 3. Soundproofing press dies.

Where: 1 - coating the bottom of the tool; 2 - movable screen of the treatment zone; 3 - covering the upper part of the instrument. Dansk Pressfabrik (Denmark) produced a KON / 100/1150 submachine gun whose sound level is $15 \%$ lower than a conventional submachine gun, which is achieved by using soundproof doors in front of a double-walled stamp and widespread use vibration-absorbing materials and soundproof materials on resonating surfaces. Hammer Maschinen GmвH (Germany) uses separate casings on highspeed die-cutting machines to cover noise sources. The walls of the casings are made of steel sheets $2 \mathrm{~mm}$ thick, coated with a layer of soundproofing material $3 \mathrm{~mm}$ thick, to which a plate of mineral wool $3 \mathrm{~mm}$ thick is glued; the entire casing is assembled in one piece by means of a soldered perforated sheet.

\section{Discussion}

Coating press guards with plates of sound-absorbing material reduces the noise level of 3 dB A. Firm I. R. Udal (England) redesigned the press guards with a force of $200 \mathrm{tf}$ so that the front and rear grilles, when lowering the slide, first move upwards, and then they turn to the press frame, closing the stamp and the working area at the moment the slide ends. The grilles are covered with sound-absorbing material, and, in addition, the fence drive mechanism is also covered by it. The same company made movable guards of the Fastrip 3 model for an eccentric press with a force of $40 \mathrm{tf}$ with a panel that can be moved vertically by means of a pneumatic drive and a fastrip of the Fastrip $3 \mathrm{~W}$ model, which tilts to the side for a press with a force of 100 te with an open frame. With the enclosure closed, noise from the treatment area is significantly reduced.

The noise level on a hammer with a separate bed with an eccentric shock is $10 \neg-15 \mathrm{dBA}$ higher than with a central one, which is associated with the excitation of vibration of the racks by the beam through the guides. To exclude eccentric impacts, preliminary stamping operations must be performed on special equipment (for example, forging rollers), and gaskets made of pagulan type material with a thickness of $1 \mathrm{~mm}$, designed for pressure up to 10 , should be installed in the joints of guides with racks of the bed of the hammer. kgf / $\mathrm{mm} 2$ and temperature up to $80 \mathrm{C}$.

It is shown that the noise level from a hammer with a round bed profile and a structural damper built into the cavity of the bed stands is $10 \mathrm{dBA}$ lower than from a hammer with an 
open bed profile. The profile of the Shabot must be convex in order to avoid the formation of a reflected wave.

The structural damping of the bed can be increased by closing the rack profile by attaching screens with a sound-absorbing coating (for example, from a layer of mineral wool) on it and installing partitions inside the closed cavities with filling the cells between the partitions with sand, gravel or shot.

To combat noise and vibration, both general and individual protective equipment is used. When planning production facilities, such as an engine test station, thermal and blacksmith shops are located on the leeward side in relation to other buildings and a residential area.

To attenuate the noise penetrating outward, it is necessary to use sound insulation of building envelopes. The rationalization of technological processes, the use of silencers, the careful fit of all moving parts of mechanisms - all this many times reduces noise. The greatest effect is achieved by replacing noisy jobs with less noisy ones.

Pneumatic riveting of frames and other parts must be replaced by hydraulic riveting or welding, forging and stamping by pressing.

To reduce vibration, it is also necessary to use special sound-absorbing structures near sources of noise or a workplace, enclose noisy units of the unit in insulating covers (gear reducers, chain, belt and other gears, impact parts, engines). When processing metal rods on automatic turret machines, the safety tube must be spring loaded or a rubber jacket inserted in the hole of this tube.

In order to reduce vibration, it is recommended to use a stiff, spring-free seat in cars, as it is a good vibration damper. Vibration acts on a person through the back, pelvis, arms. Long-term operation of the car leads to fluctuations in body parts, which negatively affects the driver. To reduce vibration, machines should be installed on a foundation deepened below the foundation of the walls, insulated from the soil by air gaps, or on specially designed shock absorbers made of steel springs or made of elastic materials.

To weaken the transmission of vibrations and noise through air ducts and pipelines, they must be connected to the fans and pumps using a flexible insert made of rubberized fabric or a rubber pipe.

It is necessary to cover vibrating surfaces and equipment with vibration absorbing and damping materials (rubber, special mastics, asbestos, bitumen, Agate plastics, etc.). At the connection points of the mating parts, shock absorbing materials (rubber, cork, cardboard, asbestos, spring shock absorbers) should be used to ensure a snug fit.

Reduce vibration in the vibration source, i.e. in the source of its formation, the following methods are possible: by excluding the impact interaction of the parts from the structure, by replacing the reciprocating movement of the parts by the rotational one, by eliminating the imbalance of rotating parts and machine components.

When working with pneumatic and electric manual machines, vibration occurs, transmitted through the handles and cases to the hands of workers, and sometimes to the legs through the medium being processed, usually when working with rammers and vibrators. To reduce vibration in this case, use handles with vibration damping or automation devices.

Personal protective equipment against vibration is used when other means are ineffective.

Shoes with shock-absorbing soles, handguards with vibration-absorbing elastic gaskets, etc. are used as personal protective equipment against vibration.

\section{Acknowledgements}

The study had no sponsorship. 


\section{References}

1. A.T. Rybak, V.P. Zharov, A.V. Serdyukov, Russian Engineering Research 29(2), 194197 (2009)

2. A. Rybak, O. Lyakhnitskaya, MATEC Web of Conferences 132, 01001 (2017)

3. A.T. Rybak, A.R. Temirkanov, O.V. Lyakhnitskaya, Russian Engineering Research 38(9), 702-704 (2018)

4. A.T. Rybak, A.R. Temirkanov, O.V. Lyakhnitskaya, Russian Engineering Research 38(3), 212-217 (2018)

5. K. Kobzev, S. Shamshura, A. Chukarin, V. Bogdanovich, V. Kasyanov, MATEC Web of Conferences 226, 01022 (2018)

6. K. Kobzev, S. Shamshura, A. Chukarin, A. Buryanovand, V. Kasyanov, MATEC Web of Conferences 226, 01023 (2018)

7. K. Kobzev, A. Chukarin, IOP Conference Series: Earth and Environmental Science 403, 012145 (2019)

8. K.O. Kobzev, E.S. Bozhko, A.V. Mozgovoi, M.D. Molev, N.I. Stuzhenko, IOP Conference Series: Materials Science and Engineering 680, 012014 (2019)

9. K.O. Kobzev, E.S. Bozhko, A.V. Mozgovoi, E.I. Kostromina, L.G. Babenko, IOP Conference Series: Materials Science and Engineering 680, 012013 (2019)

10. N. Kobzeva, V. Terentev, I. Zolotuhina, IOP Conference Series: Materials Science and Engineering 680, 012046 (2019)

11. N. Kobzeva, V. Terentev, I. Zolotuhina, IOP Conference Series: Materials Science and Engineering 680, 012011 (2019)

12. E.V. Omelchenko, E.A. Trushkova, M.V. Sidelnikov, S.L. Pushenko, E.V. Staseva, IOP Conference Series, Earth and Environmental Science Current Problems and Solutions, Ecology and Safety in the Technosphere: Current Problems and Solutions, 012018 (2017) 